\title{
EVALUATION OF TRACKING RELIABILITY METRICS BASED ON INFORMATION THEORY AND NORMALIZED CORRELATION
}

E. Loutas

\author{
Department of Informatics \\ University of Thessaloniki \\ Box 451, Thessaloniki 54124 \\ GREECE \\ E-mail: \{eloutas,nikolaid,pitas\}@ zeus.csd.auth.gr
}

\author{
I. Pitas
}


respectively and $\mathbf{x}_{k, l}^{r} \in S_{1}, \mathbf{x}_{k, l}^{c} \in S_{2}$. The mutual information of two random variables $U, V$ with a joint probability mass function $p(u, v)$ is defined as [14]:

$$
I(U, V)=\sum_{i=1}^{N_{\max }} \sum_{j=1}^{N_{\max }} p\left(u_{i}, v_{j}\right) \log _{2} \frac{p\left(u_{i}, v_{j}\right)}{p\left(u_{i}\right) p\left(v_{j}\right)},
$$

where $N_{\max }$ is the maximum number of the available grayscale levels. The probability mass functions $p(u), p(v)$ and $p(u, v)$ are empirically determined by obtaining the histograms of the grayscale values of the sets $S_{1}$ and $S_{2}$. In order to take into account the lost feature points during the tracking process a cost function $E_{m}$ is defined:

$$
E_{m}\left(U, V, N_{T}, M_{2}\right)=c_{1}\left(\frac{I(U, V)}{I_{\max }(U, V)}-\lambda_{1} \frac{N_{T}-M_{2}}{N_{T}}+c_{2}\right)
$$

The term $\frac{I(U, V)}{I_{\max }(U, V)}$ is the mutual information part of the cost function. The maximum mutual information $I_{\max }(U, V)$ is [15]:

$$
I_{\max }(U, V)=-\sum_{i=1}^{N_{\max }} p\left(u_{i}\right) \log _{2} p\left(u_{i}\right)
$$

The term $\frac{N_{T}-M_{2}}{N_{T}}$ is a penalizing quantity depending on the number of the lost feature points during the tracking process. The constants $c_{1}=0.5, c_{2}=1, \lambda_{1}=1$ are set in order to ensure that:

$$
0 \leq E_{m} \leq 1 \text {. }
$$

In the case of total occlusion: $p(v)$ and $p(u, v)$ cannot be calculated since $S_{2}=\varnothing$ and $\frac{I(U, V)}{I_{\max }(U, V)}$ is set to 0 therefore:

$$
\frac{I(U, V)}{I_{\max }(U, V)}=0 \quad \text { and } \quad \frac{N_{T}-M_{2}}{N_{T}}=1
$$

since $M_{2}=0$ leading to the minimum value of $E_{m}$. The maximum value of $E_{m}$ occurs when:

$$
I(U, V)=I_{\max }(U, V) \quad \text { and } \quad N_{T}=M_{2}
$$

\section{KULLBACK-LEIBLER DISTANCE BASED TRACKING} METRIC

The Kullback-Leibler distance is defined as [8]:

$$
D(p(u) \| p(v))=\sum_{i=1}^{N_{\max }} p\left(u_{i}\right) \log _{2} \frac{p\left(u_{i}\right)}{p\left(v_{i}\right)}
$$

and measures the similarity between $p\left(u_{i}\right)$ and $p\left(v_{i}\right)$. It is always non negative and is not symmetric [10], i.e. in general:

$$
D(p(u) \| p(v)) \neq D(p(v) \| p(u)) .
$$

The Kullback-Leibler distance can be symmetrized [10]. A symmetrical form of the Kullback-Leibler distance can be provided as

$$
\begin{gathered}
D(p(u) \| p(v))_{s_{1}}=\frac{1}{2}(D(p(u) \| p(v))+D(p(v) \| p(u))) \\
D(p(u) \| p(v))_{s_{2}}=\sqrt{D(p(u) \| p(v)) D(p(v) \| p(u))}
\end{gathered}
$$

or

$$
D(p(u) \| p(v))_{s_{3}}=\frac{D(p(u) \| p(v)) D(p(v) \| p(u))}{(D(p(u) \| p(v))+D(p(v) \| p(u)))}
$$

Therefore a variety of Kullback-Leibler-based metrics can be derived. An upper bound for the metric in equation (9) can be easily found as follows, since:

$D(p(u) \| p(v))=\sum_{i=1}^{N_{\max }} p\left(u_{i}\right) \log _{2} p\left(u_{i}\right)-\sum_{i=1}^{N_{\max }} p\left(u_{i}\right) \log _{2} p\left(v_{i}\right)$

The first term is negative or zero, while the second is positive. Therefore, an upper bound of the Kullback-Leibler distance (9) is:

$$
D(p(u) \| p(v))_{\max } \leq-\sum_{i=1}^{N_{\max }} p\left(u_{i}\right) \log _{2} p\left(v_{i}\right) .
$$

The upper bounds of the two forms of the symmetrized KullbackLeibler distance provided are:

$D(p(u) \| p(v))_{s_{1_{\max }}}=\frac{1}{2}\left(D(p(u) \| p(v))_{\max }+D(p(v) \| p(u))_{\max }\right)$

for equation (11) and

$D(p(u) \| p(v)))_{s_{2_{\max }}}=\sqrt{D(p(u) \| p(v))_{\max } D(p(v) \| p(u))_{\max }}$.

for equation (12)

A similar metric to $E_{m}\left(U, V, N_{T}, M_{2}\right)$ based on the KullbackLeibler distance can be defined as:

$$
E_{K}=c_{1}\left(1-\frac{D(p(u) \| p(v))}{D_{\max }(p(u) \| p(v))}-\lambda_{1} \frac{N_{T}-M_{2}}{N_{T}}+c_{2}\right)
$$

and by construction is expected to behave similarly to $E_{m}$. In the context of present work $c_{1}=0.5, c_{2}=1, \lambda_{1}=1$. Large values of $E_{K}$ imply a better matching between the reference and the current region.

In order to obtain a metric for the symmetrical form of equation (13) we use the values of $D(p(u) \| p(v))$ and

$D(p(v) \| p(u))$, normalized with respect to their maximum values. Therefore equation (18) will be in this case replaced with:

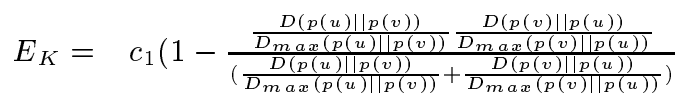

$$
\begin{aligned}
& \left.-\lambda_{1} \frac{N_{T}-M_{2}}{N_{T}}+c_{2}\right)
\end{aligned}
$$

\section{NORMALIZED CORRELATION BASED METRIC}

Normalized correlation is defined as [12]:

$$
\text { Cor }=\frac{\sum_{i=1}^{W} \sum_{j=1}^{N_{k}} J_{r}\left(\mathbf{x}_{i, j}^{r}\right) J_{c}\left(\mathbf{x}_{i, j}^{c}\right)}{\sqrt{\sum_{i=1}^{W} \sum_{j=1}^{N_{k}} J_{r}^{2}\left(\mathbf{x}_{i, j}^{r}\right) \sum_{i=1}^{W} \sum_{j=1}^{N_{k}} J_{c}^{2}\left(\mathbf{x}_{i, j}^{c}\right)}},
$$

where

$$
N_{k}=\min \left(M_{1}, M_{2}\right) \text {, }
$$


expresses the similarity between $J_{r}$ and $J_{c}$ and can be used to construct a metric similar to those already presented in Eq. (4) ,(18). The metric constructed is of the form:

$$
\text { Corr }=c_{1}\left(\text { Cor }-\lambda_{1} \frac{N_{T}-M_{2}}{N_{T}}+c_{2}\right)
$$

and was also tested under similar tracking conditions with the other two metrics. It stands that:

$$
0 \leq \operatorname{Corr} \leq 1
$$

if the values of the constants $c_{1}, c_{2}, \lambda_{1}$ are appropriately chosen. In the context of present work $c_{1}=0.5, c_{2}=1, \lambda_{1}=1$.

\section{EXPERIMENTAL RESULTS}

The above metrics were tested using the object tracking algorithm presented in [13]. Image sequences containing total occlusion and severe partial occlusion were used. Curves showing the variations of metrics $E_{m}$ and $E_{k}$ and $C o r r$ during the tracking process were acquired for different occlusion situations.

Tracking results on the football image sequence, an artificial image sequence and a head and shoulders image sequence containing partial occlusion are presented in Figures 1,2 and 3 respectively. The variations of the metric $E_{m}$ for the three sequences are presented in Figure 4, while the metric $E_{k}$ variations are presented in Figure 5. The symmetric version of equation (13) was used. Finally, the variations of the Corr metric are presented in Figure 6 . As it can be seen, metric $E_{k}$ performs similarly to $E_{m}$. Further tests on the assymetric version have shown that no significant change in $E_{k}$ behavior was caused by its asymmetry. The normalized correlation based metric Corr does not behave as well as the information theory based metrics, especially in partial occlusion situations. More specifically, in partial occlusion, Corr exhibits an abnormal behavior as can be seen in Figure 6. It should be also noted that, in the cases of the football and the artificial image sequences, feature point regeneration occurs after object reappearance. This is not the case in the head and shoulders image sequence. This difference is shown in the metric curves. The metrics $E_{m}$ and $E_{k}$ increase after the object reappearance in the football and the artificial image sequences, but their values remain low after object reappearance in the head and shoulders sequence.

\section{CONCLUSIONS}

In this paper, the performance of three different tracker reliability metrics was assessed. Two of the metrics are based on information theory, while the third is based on normalized correlation.

Experimental results have shown that the information theory based metrics behave better in partial occlusion situations than the normalized correlation based metric. A clear distinction in performance between the two information theory based metrics cannot be easily extracted. The Kullback-Leibler distance seems to provide more smooth curves. Nevertheless, the mutual information has the advantage of being unique and symmetrical.

\section{REFERENCES}

[1] T. Schoepflin, V. Chalana, D. R. Haynor, and Y. Kim, "Video object tracking with a sequential hierarchy of template deformations," IEEE Transactions on Circuits and Systems for Video Technology, vol. 11, no. 11, pp. 1171-1182, 2001.
[2] C. Erdem, A. M. Tekalp, and B. Sankur, "Metrics for performance evaluation of video object segmentation and tracking without ground trouth," in Proc. of 2001 Int. Conf. on Image Processing, 2001, vol. II, pp. 69-72.

[3] C. E. Erdem, A. M. Tekalp, and B. Sankur, "Video object tracking with feedback of performance measures," IEEE Transactions on Circuits and Systems for Video Technology, vol. 13, no. 4, 2003.

[4] K. Nickels and S. Hutchinson, "Estimating uncertainty in ssd-based feature tracking," Image and Vision Computing, vol. 20, pp. 47-58, 2002.

[5] P. Anandan, Measuring Visual Motion From Image Sequences, Phd dissertation University of Massachusetts at Amherst, 1987.

[6] C. Tomasi and T. Kanade, Shape and Motion from Image Streams: a Factorization Method - Part 3 Detection and Tracking of Point Features, Technical. report CMU-CS-91132, Computer Science Department, Carnegie Mellon University, 1991.

[7] E. Loutas, C. Nikou, and I. Pitas, "Information theory-based analysis of partial and total occlusion in object tracking," in Proc. of 2002 Int. Conf. on Image Processing, 2002, vol. II, pp. 309-312.

[8] M. N. Do and M. Vetterli, "Texture similarity measurement using kullback-leibler distance on wavelet subbands," in Proc. of 2000 Int. Conf. on Image Processing, 2000.

[9] C. Liu and H-Y Shum, "Kullback-leibler boosting," in IEEE Conference on Computer Vision and Pattern Recognition, (CVPR '03)., 2003.

[10] R. Veldhuis, "The centroid of symmetrical kullback-leibler distance," IEEE Signal Processing Letters, vol. 9, no. 3, 2002.

[11] M. N. Do, "Fast approximation of kullback-leibler distance for dependence trees and hidden markov models," IEEE Signal Processing Letters, vol. 10, no. 4, 2003.

[12] A. Papoulis, Probability, Random Variables, and Stochastic processes., Mc Graw-Hill, Inc, 1991.

[13] E. Loutas, K. Diamantaras, and I. Pitas, "Occlusion resistant object tracking," in Proc. of 2001 Int. Conf. on Image Processing, 2001, vol. II, pp. 65-68.

[14] S. Haykin, Communication Systems-3rd ed., J. Wiley, 1994.

[15] M. Skouson, Q. Guo, and Z. Liang, "A bound on mutual information for image registration," IEEE Transactions on Medical Imaging, vol. 20, no. 8, pp. 843-846, 2001. 


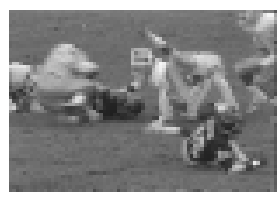

(a)

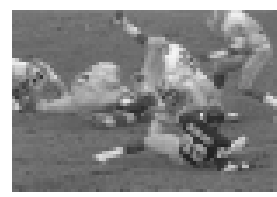

(b)

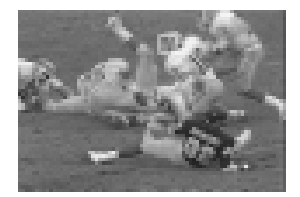

(c)

Fig. 1. Football image sequence. Tracking of the head of the football player: (a) before total occlusion, (b) total occlusion, (c) region reappearance.

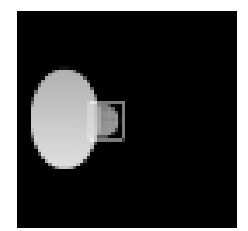

(a)

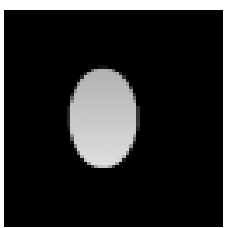

(b)

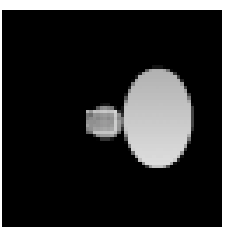

(c)

Fig. 2. Artificial image sequence: (a) before total occlusion, (b) during total occlusion, (c) region reappearance.

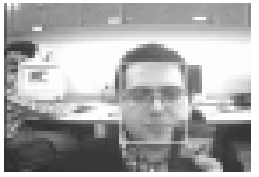

(a)

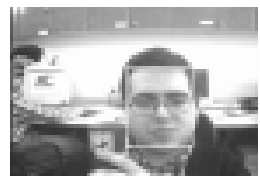

(b)

Fig. 3. Head and shoulders image sequence: (a) beginning of partial occlusion (frame 34), (b) disocclusion (frame 101).

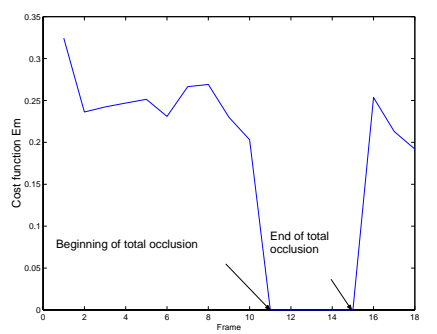

(a)

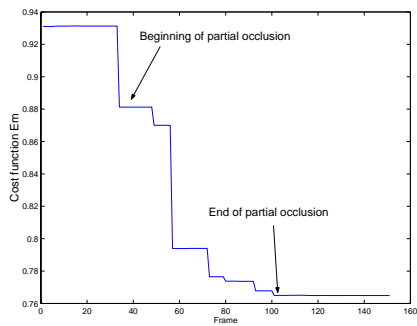

(c)

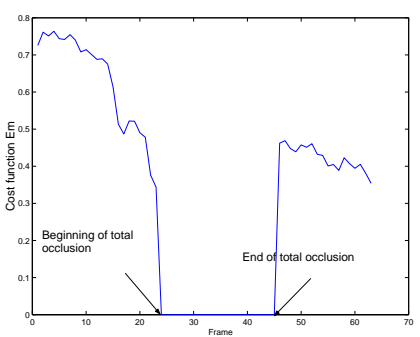

(b)

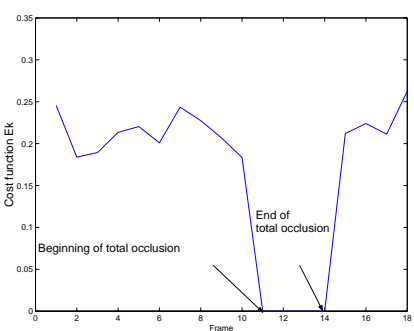

(a)

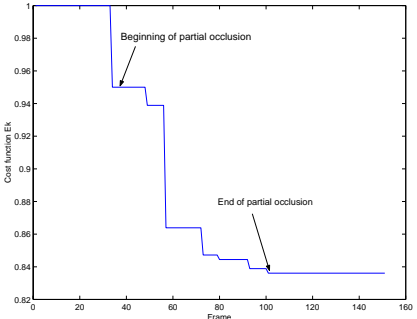

(c)

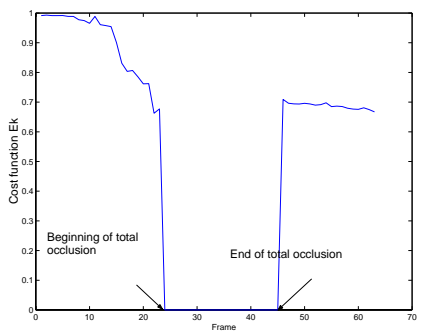

(b)

Fig. 5. (a) Values of the cost function $E_{K}$ versus frame number for part of the football image sequence (Fig. 1). Beginning of partial occlusion: frame 10. Beginning of total occlusion: frame 11. End of total occlusion: frame 14.(b) Cost function $E_{K}$ for the artificial image sequence (Fig. 2) versus frame number. Beginning of partial occlusion: frame 16. Beginning of total occlusion: frame 24. End of total occlusion: frame 45. (c) Values of the cost function $E_{K}$ versus frame number for the head and shoulders image sequence (Fig. 3). Beginning of partial occlusion: frame 34. End of partial occlusion: frame 101 .

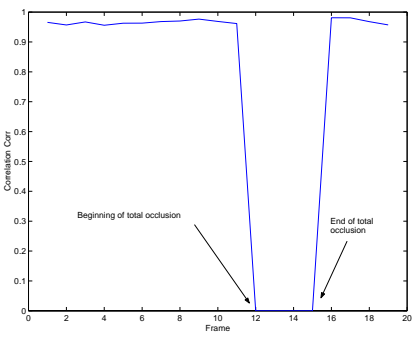

(a)

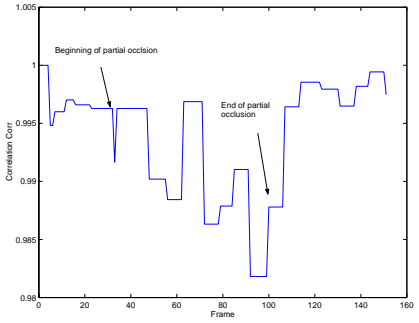

(c)

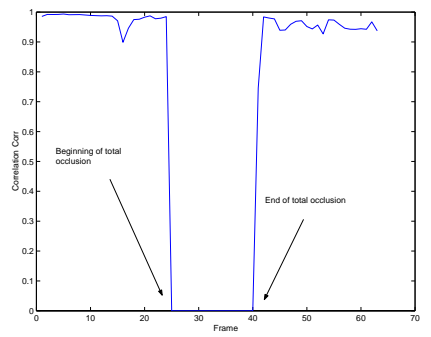

(b)

Fig. 6. (a) Normalized Correlation for the football image sequence (Fig. 1) versus frame number. Beginning of partial occlusion: frame 10. Beginning of total occlusion: frame 12. End of total occlusion: frame 15.(b) Normalized Correlation for the artificial image sequence (Fig. 2) versus frame number. Beginning of partial occlusion: frame 16. Beginning of total occlusion: frame 25 . End of total occlusion: frame 45. (c) Normalized Correlation for the head and shoulders image sequence (Fig. 3) versus frame number. Beginning of partial occlusion: frame 34 . End of partial occlusion: frame 101. 